\title{
Synergistic action of bacteriophage with antibiotics on Pseudomonas aeruginosa biofilms
}

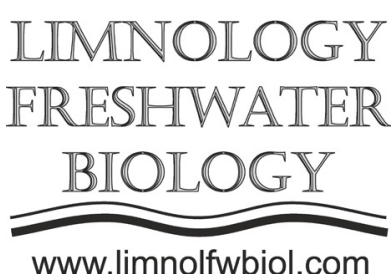

\author{
Sabirova A.E. ${ }^{1 *}$, Gorshkova A.S. ${ }^{2}$, Drucker V.V. ${ }^{2}$, Kayumov A.R. ${ }^{1}$ \\ ${ }^{1}$ Kazan Federal University, Kremlevskaya Str., 18, Kazan, 420008, Russia \\ ${ }^{2}$ Limnological Institute of the Siberian Branch of the Russian Academy of Sciences, Ulan Batorskaya Str., 3, Irkutsk, 664033, Russia
}

\begin{abstract}
An opportunistic microorganism Pseudomonas aeruginosa causes nosocomial infec tions and often forms rigid biofilms on both intact human skin and wounds, as well as the surfaces of artificial implants, prostheses and catheters. Being embedded into the exopolymeric matrix of the biofilm bacteria are extremely resistant to antibiotics, biocides, and the immune system of the human. Therefore, the search for new approaches for eradication of bacterial biofilm is an urgent challenge of modern medicine. Combined therapy of biofilms with antibiotics and phages has shown promising results. In this study, 48-hour monomicrobial biofilms of Pseudomonas aeruginosa were treated with phage from the family Myoviridae isolated from Lake Baikal and 12 antibiotics. While the treatment with a single phage did not significantly affect neither planktonic nor biofilm-embedded bacteria, in the presence of the phage, the MICs of gentamicin, ciprofloxacin, imipenem and meropenem the biofilm decreased 4-8-fold. Moreover, the density of biofilm was also significantly dropped in compare with the treatment by solely antibiotics or phage.
\end{abstract}

Keywords: Pseudomonas aeruginosa, microbial biofilms, bacteriophages, antibiotics, synergism

\section{Introduction}

Biofilm formation is believed as one of the main factors leading to chronic $P$. aeruginosa infections (Bjarnsholt, 2013). Biofilms are communities of microbial cells immersed into the polymeric matrix formed on the surface of tissues, medical devices, catheters, and plastic implants. The bacteria in the biofilm are highly resistant to biocides, antibiotics and the human immune system, causing chronic reinfections and inflammatory complications in the postoperative period (Hilbert and Moore, 2005).

Bacteriophages (or phages), viruses infecting the bacteria, are natural antibacterial agents that specifically target and lyse bacteria. Some phages can penetrate the inner layers of biofilms and infect dormant cells (Pires et al., 2017), which is a clear advantage of phages over antibiotics in treatment of biofilms. Some studies have shown the efficiency of antibiotics combinations with phages on liquid cultures and biofilms of $P$. aeruginosa (Chaudhry et al., 2017). The advantage of this approach is 1) the low possibility of simultaneous development of resistance to both agents and 2) the effective concentrations of antibacterial agents are decreased several fold providing lowered risks of side effects.

\section{Materials and methods}

Pseudomonas aeruginosa ATCC ${ }^{\circledR} 27853^{\text {тм }}$ and the bacteriophage belonging to Myoviridae family, isolated from Lake Baikal and termed PaSA1 were used in this study. To analyze the biofilm, the bacteria were grown under static conditions at $35^{\circ} \mathrm{C}$ for 48 hours in BM broth (Kayumov et al., 2015; Baidamshina et al., 2017). Biofilm formation was evaluated in 24well polystyrene plates (Eppendorf) by staining with crystal violet (Sharafutdinov et al., 2017). The minimal inhibitory concentration (MIC) of antimicrobials was determined by broth microdilution in accordance with the EUCAST recommendation. Cell viability was assessed using confocal laser scanning microscopy and a resasurine assay.

\section{Results and discussion}

Among various bacteriophages from Lake Baikal in the area of wastewaters shedding close to Slyudyanka city, the isolate PaSA1 was capable of lysing $P$. aeruginosa ATCC 27853 cells. By using the transmission electron microscope the phage has been identified according to morphology as a member of the Myoviridae family. Next, the synergistic effect of the bacteriophage with 12 different antibiotics was studied: gentamicin, amikacin,

*Corresponding author.

E-mail address: alinka.zam@mail.ru (A.E.Sabirova)

(C) Author(s) 2020. This work is distributed under the Creative Commons Attribution 4.0 License. 
ciprofloxacin, norfloxacin, ceftriaxone, streptomycin, erythromycin, azithromycin, vancomycin, imipenem, meropenem and colistin. In combination with phage, the MIC of gentamicin, ciprofloxacin, imipenem and meropenem were 4-8 times lower in compare with solely antibiotic. The synergistic effect of these drugs and the phage was also observed on 48-h old biofilms. Taken together, our data allow suggesting PaSA1 as promising tool for enhancement of antimicrobial efficacy against $P$. aeruginosa including its biofilms.

\section{Acknowledgments}

This work was supported by the Russian Foundation for Basic Research, grant No 20-04-00247 and Russian Government Program of Competitive Development of Kazan Federal University. The microscopy has performed by using facilities of Centre "Ultramicroanalysis" of Limnological Institute of the Siberian Branch of the Russian Academy of Sciences.

\section{References}

Baidamshina D.R., Trizna E.Y., Holyavka M.G. et al. 2017. Targeting microbial biofilms using Ficin, a nonspecific plant protease. Scientific Reports 7. DOI: 10.1038/srep46068
Bassetti M., Vena A., Croxatto A. et al. 2013. How to manage Pseudomonas aeruginosa infections. Drugs Context 7. DOI: $10.7573 /$ dic. 212527

Bjarnsholt T. 2013. The role of bacterial biofilms in chronic infections. APMIS 136: 1-51. DOI: 10.1111/ apm.12099

Chaudhry W.N., Concepcion-Acevedo J., Park T. et al. 2017. Synergy and order effects of antibiotics and phages in killing Pseudomonas aeruginosa biofilms. PLoS ONE 12. DOI: 10.1371/journal.pone.0168615

Hilbert P., Moore L.E. 2005. Cationic antiseptics: a variety of actions under a common epithet. Journal of Applied Microbiology 4: 703-715. DOI: 10.1111/j.1365-2672.2005.02664.x

Kayumov A.R., Khakimullina E.N., Sharafutdinov I.S. et al. 2015. Inhibition of biofilm formation in Bacillus subtilis by new halogenated furanones. Journal of Antibiotics 68: 297-301. DOI: 10.1038/ja.2014.143

Pires D.P., Melo L.D.R., Vilas Boas D. et al. 2017. Phage therapy as an alternative or complementary strategy to prevent and control biofilm-related infections. Current Opinion in Microbiology 39: 48-56. DOI: 10.1016/j.mib.2017.09.004

Sharafutdinov I.S., Trizna E.Y., Baidamshina D.R. et al. 2017. Antimicrobial effects of sulfonyl derivative of $25 \mathrm{H}$-furanone against planktonic and biofilm associated methicillin-resistant and -susceptible Staphylococcus aureus. Frontiers in Microbiology 8. DOI: 10.3389/fmicb.2017.02246 